\title{
Application of silk fibroin/ chitosan wound dressing to rat skin regeneration
}

\author{
Zhi-hua $\mathrm{Lu}^{1, \mathrm{a} *}$, Yu-dong Ma ${ }^{1, \mathrm{~b}}$, Dong-mei Zhao ${ }^{2, \mathrm{c}}$, Hong-yao $\mathrm{Xu}^{3, \mathrm{~d}}$ \\ ${ }^{1}$ Department of Physics and Information Engineering, Jining University, Qufu 273155, PR China \\ ${ }^{2}$ The Second Hospital of Shandong University, Jinan 250033, PR China \\ ${ }^{3}$ College of Material Science and Engineering, Donghua University, Shanghai, 201620 , PR China \\ a zlh_jeeps@163.com, ${ }^{b}$ mayudong0620@163.com, \\ c dongmeizhao663@163.com,d hongyaoxu@dhu.edu.cn
}

\begin{abstract}
Keywords: silk fibroin; chitosan; wound dressing; skin regeneration
ABSTRACT: In this study, a new bi-layered silk fibroin (SF)/ chitosan (CS) wound dressing was investigated. The morphology, cytocompatibility and effect on open wound healing in rats were studied. The results show that the SF/CS wound dressing has homogeneous porous structure and support the attachment and proliferation of L929 mouse fibroblasts. Furthermore, it proves that, comparing with the clinically used wound dressing, the SF/CS wound dressing promote skin healing in full-thickness wounds, and show greater extent in wound size reduction, epitheliazation and collagen formation. The empirical results in this study indicate that SF/CS wound dressing maybe a good candidate for skin regeneration.
\end{abstract}

\section{INTRODUCTION}

Skin plays an important role in homeostasis and the prevention of invasion by microorganisms. Generally, skin should to be covered with a dressing immediately after it is injured ${ }^{[1,2]}$. An ideal wound dressing is usually made of available biomaterials that have no allergies, nontoxic, antimicrobial, as well as promote wound healing ${ }^{[3,4]}$. From the various studies reported in literature, both silk fibroin ${ }^{[5-7]}$ and chitosan ${ }^{[8,9]}$ are natural biocompatible materials, which seemed to be excellent for wound healing. In our previous work, a novel SF/ CS wound dressing with excellent mechanical properties and antibacterial performance was synthesized. Briefly, Silk was first purified from its sericin content, and then weaved to obtain silk fibroin fabric by ring spinning. After that, chitosan was sprayed on the fabric to obtain a bi-layered SF/CS wound dressing. In the present study, we have attempted to further detect the functional properties of the dressing.

\section{EXPERIMENTAL}

\section{Materials}

SF/ CS wound dressing was supplied by Professor Hongyao Xu, Donghua University, China. Mouse fibroblasts (L929) were obtained from Shanghai Saiqi Biological Technology Co., Ltd., China. RPMI 1640 medium, 1\% Penicillin-streptomycin, trypsin, 10\% fetal bovine serum and MTT powder were supplied by Shanghaikayon Biological Technology Co., Ltd., China. Other reagents were all A.R. grade, and used without further purification.

\section{Characterization of SF/CS wound dressing}

The morphology of the SF/CS wound dressing was determined by scanning electron microscope (sigma, Carl Zeiss, Germany) at accelerating voltage of $5 \mathrm{kV}$. The cytotoxicity of the dressing was examined by MTT assay; the morphology and density of L929 cells cultured in the samples for 5 days were observed.

\section{Open wound-healing test}

Sprague-Dawley rats (28-week-old, 250 $\pm 5 \mathrm{~g}, \mathrm{n}=24)$ were used in this study. After 
anesthetization, the full-thickness wounds $(1.2 \mathrm{~cm} \times 1.2 \mathrm{~cm})$ were prepared on both left and right sides of their back (2 wounds/ rat). The bi-layered SF/CS wound dressing was then applied to the wounds of each rat, while a commercial wound dressing material " $3 \mathrm{M}^{\mathrm{TM}}$ Tegaderm high performance foam adhesive dressing (3M Corporate Headquarters, MN, USA)" served as a comparison.

On the 3rd, 9th and 14th postoperative days, macroscopic photographs of the wounds were taken and the wound area was measured using an image analysis program. The tissue regenerated at the wound was collected by histological and immunohistochemical evaluation on the 3rd, 6th, and 14th postoperative days.

\section{Statistical analysis}

All the results were statistically analyzed by the unpaired student's $t$ test and $\mathrm{p}<0.05$ was considered to be statistically significant.

\section{RESULTS AND DISCUSSION}

SEM micrographs of silk fibroin fabric and SF/CS wound dressing were shown in Fig.1. It could be found that, at the microscopic magnification, smooth surface of silk fibroin fibers was observed before and after chitosan-coated; the structure of chitosan-coated silk fibroin seemed denser than that of non-coated silk fibroin, and the chitosan coating thickened the SF fibers.

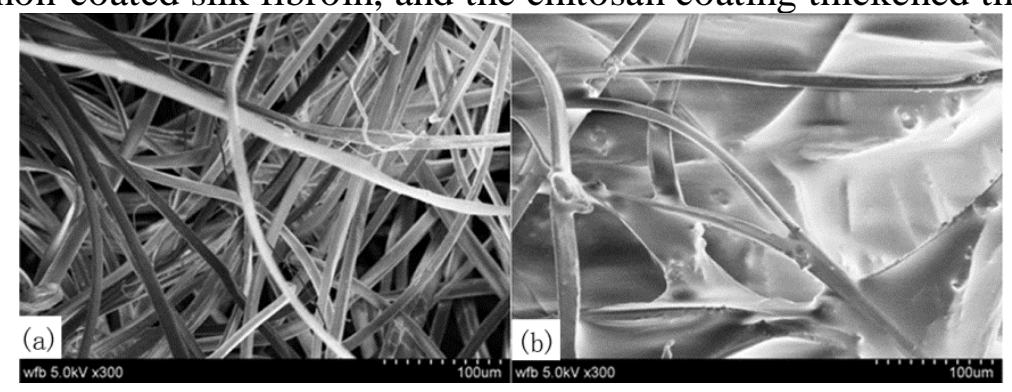

Fig.1 SEM images of silk fibroin fabric (a) and SF/CS wound dressing (b)

The initial cell adhesion and spreading are important factors in developing wound dressing for tissue engineering. The cytocompatibility of the SF/CS fabric was studied by MTT assays and the result was shown in Fig.2. The cells showed spreading morphology and proliferated homogeneously throughout the porous structure. High density of cells proliferated on the dressing was confirmed. The experimental data in Fig.2 suggested that the silk fibroin/ chitosan composite exhibited outstanding performance in cell proliferation during culture, which may be used as a good candidate for skin regeneration.

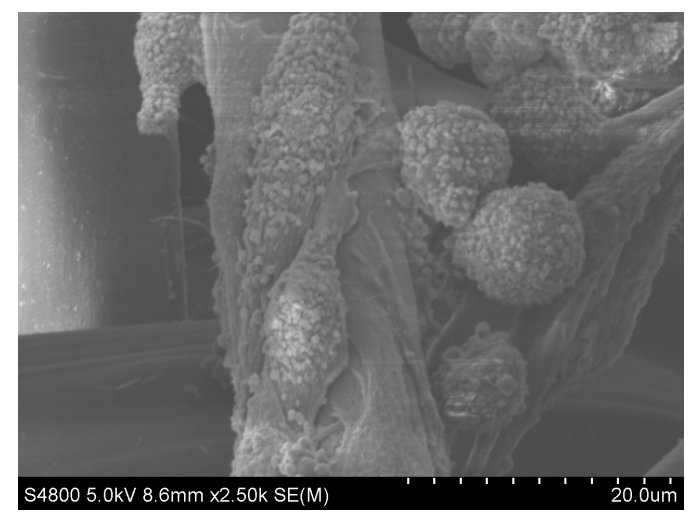

Fig.2 Microscopic images of L929 cells cultured on the SF/CS wound dressing

The macroscopic appearance and area of wounds dressed with $3 \mathrm{M}^{\mathrm{TM}}$ Tegaderm high performance foam adhesive dressing and SF/CS wound dressing at different postoperative time 
were shown in Fig.3 and Fig.4. Wound healing in control and SF/CS wound dressing were similar on the operation day and 3rd day. Wound area was reduced along the treatment time in both sides, but the area of wounds dressed with the SF/CS wound dressing seemed to be smaller than that of the $3 \mathrm{M}^{\mathrm{TM}}$ Tegaderm high performance foam adhesive dressing. SF/CS dressing was found to better than commercial dressing $(\mathrm{p}<0.05)$ in promoting wound healing, and it almost completely healed the wounds at 14th postoperative day.

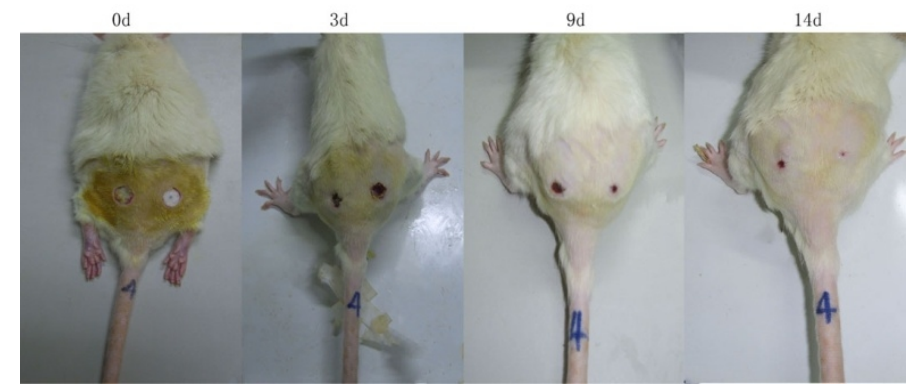

Fig. 3 Gross images of full thickness wounds dressed with SF/CS wound dressing (right) and $3 \mathrm{M}^{\mathrm{TM}}$ Tegaderm high performance foam adhesive dressing (left) after different surgery time

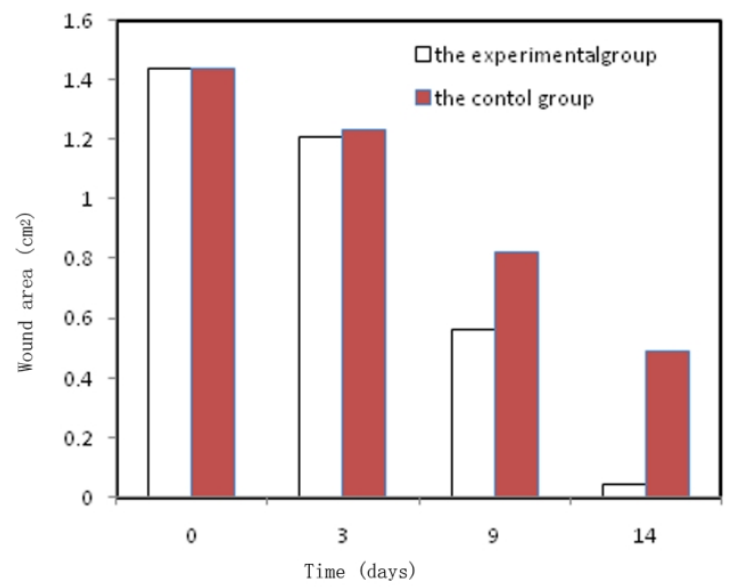

Fig.4 Area of full thickness wounds dressed with SF/CS wound dressing (the experimental group) and $3 \mathrm{M}^{\mathrm{TM}}$ Tegaderm high performance foam adhesive dressing (the control group) after different surgery time.

H\&E staining was performed to evaluate the speed and quality of the newly regenerated tissue and the results were presented in Fig.5. At 3days after operation, sparse inflammatory cells in the dermis were detected in the wound treated by SF/CS. On the contrary, the wound treated with commercial dressing showed prominent inflammatory cell infiltration. At 6 days after surgery, there was no discernible difference among histological sections of wounds treated by SF/CS and commercial dressing. The wounds dressed with SF/CS dressing showed higher degree of newly formed collagen tissue than those of commercial dressing along treatment. At 14 days after surgery, the wound treated with SF/CS was almost healed. This further testified that the SF/CS dressing could be a suitable wound dressing for skin regeneration. 


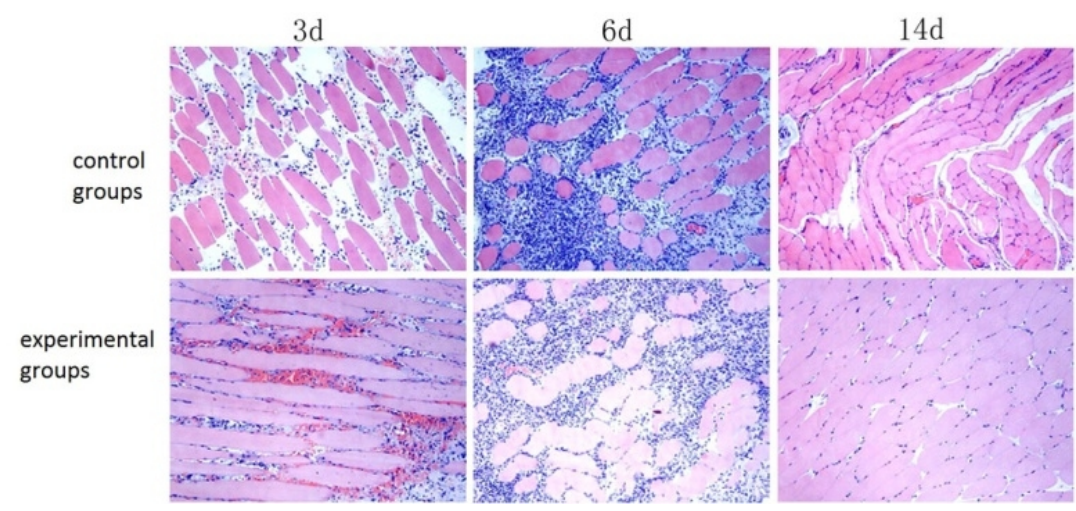

Fig.5 H\&E-staining images of full-thickness wounds dressed with SF/CS wound dressing (experimental groups) and $3 \mathrm{M}^{\mathrm{TM}}$ Tegaderm high performance foam adhesive dressing (control groups) after different surgery time

\section{CONCLUSIONS}

In this work, the bi-layered SF/CS wound dressing were prepared and characterized. SEM micrographs show that the SF/CS dressing has a uniform structure and chitosan coating thicken the SF fibers. Indirect cytotoxicity assessments of SF/CS wound dressing with L929 cells indicate it has good in vitro biocompatibility. Open wound healing test in a rat model certified SF/CS wound dressing promote the wound healing. The SF/CS wound dressing has the potential to be used for skin regeneration.

\section{ACKNOWLEDGEMENTS}

Financial support by the Natural Science Foundation of China (11347176), Shandong Excellent Young Scientist Research Award Foundation (BS2011CL010, BS2011NY003) , Shandong Province Higher School Science and Technology Plan Project (J15LA58) and Shandong province science and technology development projects(2012GGE27065) .

\section{REFERENCES}

[1] Rui Xu, Gaoxing Luo, Hesheng Xia, Weifeng He, Jian Zhao, Bo Liu, Jianglin Tan, Junyi Zhou, Daisong Liu, Yuzhen Wang, Zhihui Yao, Rixing Zhan, Sisi Yang, Jun Wu, 2015. Biomaterials 40 $1-11$.

[2] George Dan Mogosanu, Alexandru Mihai Grumezescu, 2014. Int. J. Pharm.463 127-136.

[3] Shih-Jung Liu, Yi-Chuan Kau, Chi-Yin Chou, Jan-Kan Chen, Ren-Chin Wu, Wen-Ling Yeh, 2010. J. Membrane Sci. 355 53-59.

[4] Sorada Kanokpanont, Siriporn Damrongsakkul, Juthamas Ratanavaraporn, Pornanong Aramwit, 2012. Int. J. Pharm. 436 141-153.

[5] Laura J. Bray, Karina A. George, Dietmar W. Hutmacher, Traian V. Chirila,Damien G. Harkin, 2012. Biomaterials 33 3529-3538.

[6] Andreia Vasconcelos, Andreia C. Gomes, Artur Cavaco-Paulo, 2012. Acta Biomater. 8 3049-3060.

[7] Semih Çalamak, Ceren Erdoğdu, Meral Özalp, Kezban Ulubayram, 2014. Mat. Sci. Eng. C 43 11-20.

[8] Yingshan Zhou, Hongjun Yang, Xin Liu, Jun Mao, Shaojin Gu, Weilin Xu, 2013. Int. J. Biol. Macromol. 53 88-92.

[9] R. Jayakumar, M. Prabaharan, P.T. Sudheesh Kumar, S.V. Nair, H. Tamura, 2011. Biotechnol. Adv. 29 322-33. 